\title{
Untere Mittelterrasse und Krefelder Mittelterrasse im Südteil der Niederrheinischen Bucht
}

\author{
Von Klaus-P. Winter, Frankfurt am Main
}

Mit 5 Abbildungen

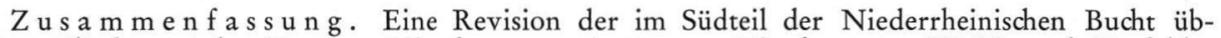
lichen Gliederung der jüngeren Mittelterrassen in Untere Mittelterrasse (UMT) und Krefelder Mittelterrasse (KMT) ergab: Der morphologisch deutlichen Zweiteilung der Terrassenflur im Raume Köln liegt keine Unterteilung der fluviatilen Aufschüttung zugrunde. Sie wird durch stark unterschiedliche Deckschichtenmächtigkeiten vorgetäuscht. Die abgedeckte Schotteroberfläche der UMT ist mit ausgeglichenem Längs- und Quergefälle über die ganze Breite dieser Talstufe von Bonn bis Stommeln (südlich Köln) zu verfolgen. Es liegen keine Hinweise auf eine noch tiefere Mittelterrasse (= KMT) vor. Der Profilaufbau des Schotterkörpers und die sedimentologische Entwicklung geben ebenfalls keine Anhaltspunkte für die bisher u. a. angenommene streckenweise Überlagerung der UMT durch Aufschüttungen der KMT.

Die für die UMT als typisch geltende interglaziale Bodenbildung wurde in allen Profilen beobachtet. Diese Ergebnisse zeigen, daß die KMT im Südteil der Niederrheinischen Bucht nicht ausgebildet ist.

S u m mary. The area of the Lower Middle-Terrace (Untere Mittelterrasse) and the Krefeld Middle-Terrace (Krefelder Mittelterrasse) in the southern part of the Lower Rhine Vallay has been restudied. Results: In the western part of Köln the morphological division of these terraces is not due to fluvial accumulation but dependent on the different thickness of overlaying deposits (loess). The Lower Middle-Terrace covers the whole vallay step. The Krefeld MiddleTerrace is not developed.
\end{abstract}

\section{Einleitung}

Trotz der in den letzten zwanzig Jahren verstärkten Anstrengungen, unter Anwendung verschiedenster Untersuchungsmethoden die Gliederung der Rheinterrassen in der Niederrheinischen Bucht zu verbessern, blieben Probleme ungeklärt bzw. nur unbefriedigend gelöst. Das gilt in besonderem Maße für die Mittelterrassen im Südteil der Niederrheinischen Bucht - hier speziell für die Beziehungen zwischen der U n t e r e n Mittelterrasse (UMT) und der Krefelder Mittelterrasse (KMT).

Nordwestlich von Köln ist neben der UMT eine weitere ausgedehnte Geländestufe ausgebildet, die von der UMT morphologisch deutlich abgesetzt ist. Sie wird seit QUITzow (1956) als der südliche Ausläufer der KMT im Raume Krefeld aufgefaßt.1) Stromaufwärts geht diese Geländestufe unter allmählicher Abnahme des vertikalen Abstandes westlich von Köln in die Terrassenflur der UMT über (Abb. 1).

Insbesondere das Problem dieses morphologischen Übergangs in die UMT gab seit Fliegel (1908) bis in die jüngste Zeit (z. B. Kopp \& Woldstedt 1965) wiederholt Anlaß zu Deutungsversuchen. So war bislang die schon von Fliegel aufgeworfene Frage, ob diese "tiefste Mittelterrassenstufe" lediglich eine Erosionsform in der UMT ist, oder ob sie eine selbständige Akkumulation darstellt, nicht entschieden.

Quitzow (1956) nimmt an, daß diese Geländestufe zwischen UMT und Niederterrasse (NT) eine selbständige Aufschüttung (= KMT) darstellt. Diese Ansicht wird auch in den

1) Die Krefelder Mittelterrasse - als jüngste Mittelterrassenstufe - besitzt ihre größte Verbreitung im Raume Krefeld. War schon ihre Abgrenzung als selbständige Terrasse gegen die UMT problematisch (vgl. hierzu u. a. Steeger 1925a, 1925b, 1926 und Bertsch, Steeger \& Steusloff 1931), so konnte ihre stratigraphische Stellung bis heute nicht eindeutig festgelegt werden (z. B. THOME 1959). 
folgenden Arbeiten von Breddin (1958), Vinken (1959), Kaiser (1961) und PaAs (1962) vertreten. Für den morphologischen Angleichungsbereich beider Terrassen und die stromaufwärts gelegenen Abschnitte der UMT ergaben sich damit zwangsläufig zwei Deutungsmöglichkeiten:

a) Die Aufschüttung der KMT hat hier gerade das Niveau der UMT erreicht,

b) die KMT hat Teile der UMT überschottert.

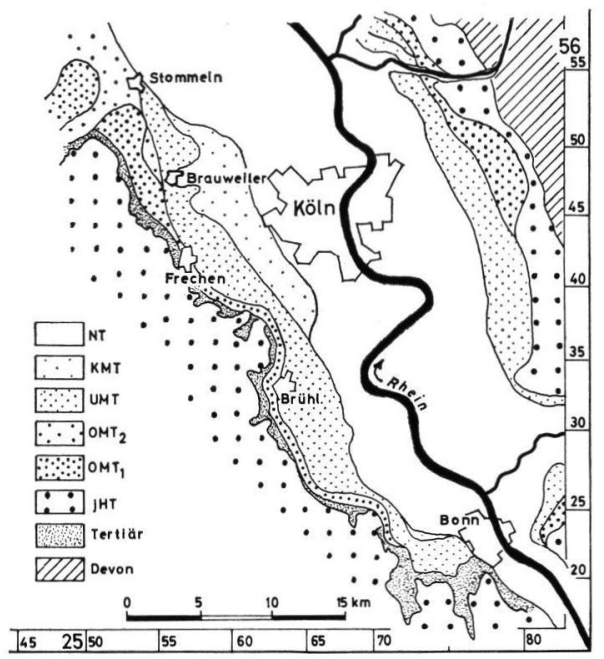

Abb. 1. Ubersichtskarte der Flußterrassen im Südteil der Niederrheinischen Bucht (nach Quirzow 1956, Kaiser 1961, Ahorner 1962, PaAs 1962 und Heller \& Brunnacker 1966).

Von Quitzow wird die zweite Möglichkeit als wahrscheinlicher angesehen. In gleichem Sinne äußert sich auch PAAS, der auf Grund pedologischer Untersuchungen eine Verbreitung des Schotterkörpers der KMT zumindest in den nahe zur Niederterrasse gelegenen Abschnitten der UMT bis in den Raum von Bonn annimmt (Abb. 1).

Eine Neubetrachtung der bisherigen Ergebnisse und Deutungen wurde ausgehend von einer morphologischen Aufnahme der Terrassenfluren der UMT und KMT und nach einer Auswertung der aus diesem Gebiet vorliegenden zahlreichen Bohrprofile möglich. Die Bohrprofile gestatteten sichere Aussagen über die Deckschichtenmächtigkeiten und damit über die Gestaltung der Schotteroberfläche. Zudem wurden die Untersuchungsergebnisse der Profilentwicklung und des sedimentologischen Aufbaus der UMT in diesem Gebiet (WINTER 1968) herangezogen.

Die Arbeiten blieben auf das linksrheinische Gebiet beschränkt, da rechtsrheinisch mit Ausnahme kleinerer, weiter nördlich gelegener Vorkommen der KMT zwischen Düsseldorf und Leverkusen (PAAs 1962) nur die UMT vertreten ist.

\section{Morphologische Situation}

Für die Untersuchungen im Bereich der UMT und KMT wurden neben der eigenen Kartierung auch die morphologischen Daten aus älteren geologischen Karten herangezogen, da die ursprüngliche Geländeform besonders im morphologischen Übergangsbereich UMT/KMT durch dichtere Besiedlung heute kaum noch in ihren Zusammenhängen zu erkennen ist. Der betrachtete Raum zeigt zwei morphologisch unterschiedliche Bereiche - im folgenden als südlicher und nördlicher Abschnitt bezeichnet - die etwa in Höhe des Frechener Baches aneinandergrenzen (Abb. 2). 


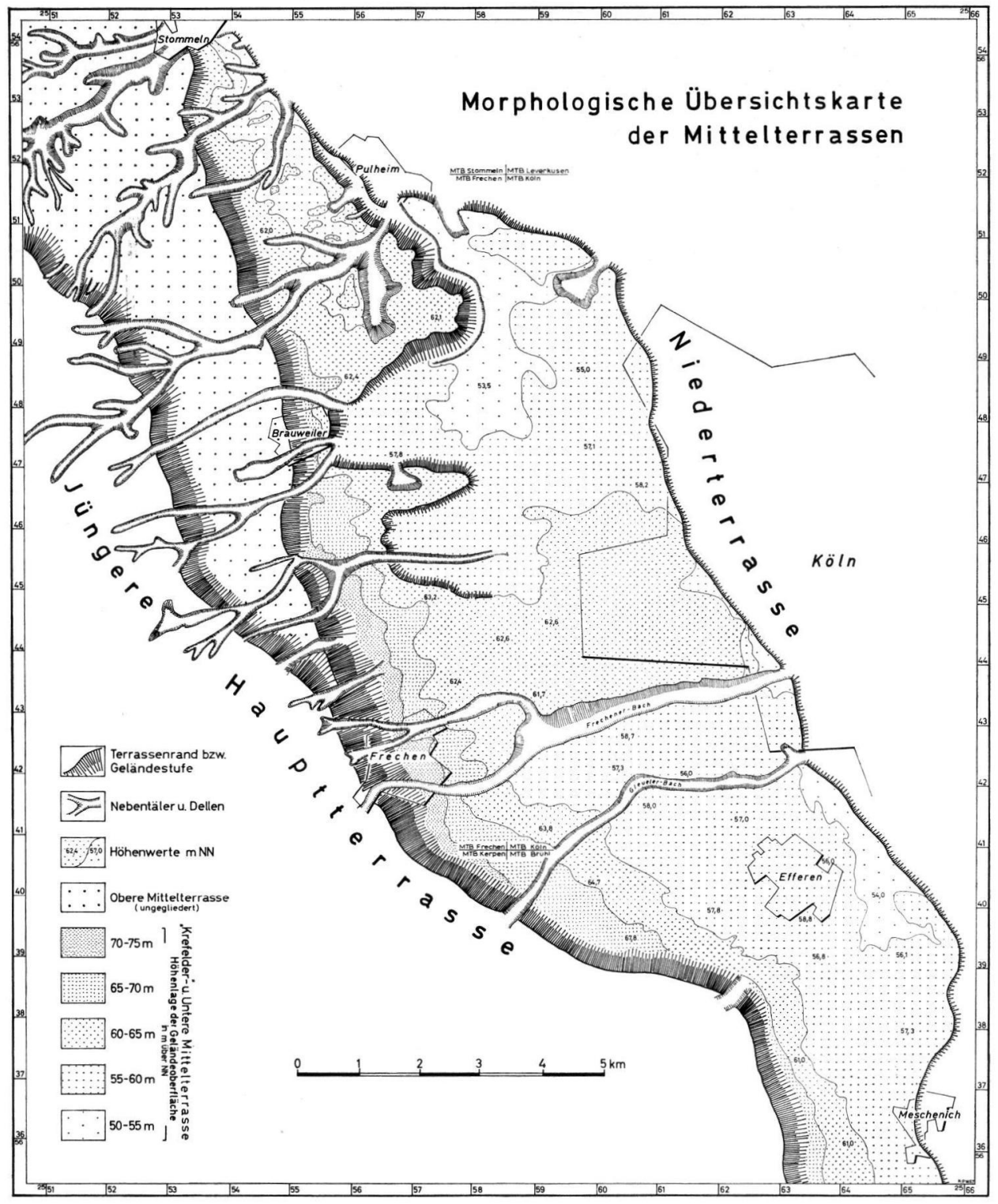

Abb. 2. Morphologische Ubersichtskarte der Mittelterrassen im Raume Köln.

\section{Südlicher Abschnitt}

Der südliche Abschnitt - also die von der UMT eingenommene Terrassenfläche zwischen Bonn und dem Frechener Bach - zeigt ein ausgeglichenes Längsgefälle von ca. $+75 \mathrm{~m}$ NN bei Bonn auf $+57 \mathrm{~m} \mathrm{NN}$ in der Höhe des Frechener Baches. Die UMT besitzt in diesem Abschnitt eine fast ebene Geländeoberfläche, die nur gelegentlich von flachen Dellen und Rinnen unterbrochen ist. Gegen die Niederterrasse ist die Terrassenflur durch einen markanten Erosionshang von 5-8 m Höhe morphologisch deutlich abgesetzt. Ihre uferwärtige Begrenzung ist in der Regel durch einen zunächst sanften, dann aber 
steilen Geländeanstieg gegen die höheren Mittelterrassen (z. B. die OMT) bzw. gegen die Jüngere Hauptterrasse ( $\mathrm{jHT}$ ) charakterisiert. Die OMT ist hier nur in schmalen Erosionsresten erhalten, die im Anstieg zur Hauptterrasse morphologisch nicht sicher zu verfolgen sind.

Unmittelbar nördlich des Frechener Baches wird das bis dahin ausgeglichene Längsgefälle der UMT von einem bisher nicht weiter beachteten, quer über die ganze Terrassenflur verlaufenden, riegelartigen Geländeanstieg von $4-5 \mathrm{~m}$ Höhe (von $+57 \mathrm{~m} \mathrm{NN}$ auf maximal $+63 \mathrm{~m} \mathrm{NN}$ ) unterbrochen (Abb. 2). Dieser Anstieg bildet die südliche Begrenzung eines $2-3 \mathrm{~km}$ breiten Geländerückens mit durchschnittlichen Höhenlagen um $+62 \mathrm{~m} \mathrm{NN}$, der in seiner Form an einen von Westen her angeschütteten Schwemmkegel erinnert. Gegen Norden erfolgt dann wieder ein ganz allmählicher, kaum wahrnehmbarer Geländeabfall. Er leitet in die bislang zur KMT gestellte Terrassenfläche über, deren Höhenwerte um $+55 \mathrm{~m}$ NN pendeln. Wir befinden uns damit bereits im nördlichen $\mathrm{Ab}-$ schnitt, in der Zone der bisher angenommenen morphologischen Differenzierung der KMT aus der UMT.

\section{Nördlicher Abschnitt}

Auch hier ist ein deutlicher Erosionshang gegen die Niederterrasse ausgebildet. Bemerkenswert ist eine stärkere Zerschneidung der Terrassenfläche, wobei die heute meist trockenen Rinnen bis in die Terrassenfluren der Hauptterrasse zurück zu verfolgen sind.

Dieser nördliche Abschnitt ist durch drei markante Geländestufen gekennzeichnet. Fliegel (1908) bezeichnete sie als „höchste, mittlere und tiefste Mittelterrassenstufe“. Diese morphologische Aufgliederung wurde in den bisherigen Arbeiten übernommen. So wurde die obere Geländestufe ( $+70-90 \mathrm{~m} \mathrm{NN}$ ) zur OMT, die mittlere Geländestufe $(+60-65 \mathrm{~m} \mathrm{NN})$ zur UMT und die untere Geländestufe $(+50-55 \mathrm{~m} \mathrm{NN})$ zur KMT gestellt.

Stromaufwärts verschmälert sich die hier nicht weiter interessierende Terrassenflur der OMT bis sie nördlich Frechen morphologisch in den Erosionshang zur Jüngeren Hauptterrasse einmündet.

In der Folge werden zum besseren Verständnis die mittlere Geländestufe als „h öh e r e Flä ch e“ (bisher als UMT aufgefaßt) und die untere Geländestufe als „t i e f e r e Fläche" (bisher als KMT aufgefaßt) bezeichnet.

Stromaufwärts gesehen zeigt sich das folgende morphologische Bild (Abb.2). In der Höhe von Pulheim setzt, von der „höheren Fläche“ durch einen markanten Geländeabfall (von +60 auf $55 \mathrm{~m} \mathrm{NN}$ ) getrennt, die „tiefere Fläche“ ein, die rasch an Breite gewinnt. Dieser Geländeabfall wurde in den bisherigen Arbeiten als Terrassenhang angesehen. Er zeigt zunächst einen über ca. $4 \mathrm{~km}$ verfolgbaren NW-SE gerichteten Verlauf parallel den Terrassenlehnen von OMT, UMT und NT. Nordöstlich von Brauweiler biegt er dann nach SW um, wodurch die Breite der „höheren Fläche“ erheblich reduziert wird und setzt sich in einem weiten Bogen bis in die Höhe von Lövenich fort. Hier schwingt der bis dahin morphologisch gut auszumachende Geländeabfall erneut zurück und läuft unter allmählicher Abnahme der Böschungshöhe in einem zweiten, enger geschwungenen Bogen in der „höheren Fläche“ aus (Abb. 2).

Betrachtet man nun das Längsgefälle der UMT im bisherigen Sinne, so fällt auf, daß nördlich des Frechener Baches von bereits erreichten $+57 \mathrm{~m}$ NN flußabwärts wieder ein Anstieg auf $+60 \mathrm{~m}$ NN festzustellen ist, und daß diese Höhe konstant über den ganzen nördlichen Bereich bis Stommeln beibehalten wird. Dagegen ist das Längsgefälle der bisher zur KMT gestellten „tieferen Fläche“ nördlich des morphologischen Überganges mit anfänglich $5 \mathrm{~m}$ Höhenverlust auf $1,5 \mathrm{~km}$ auffällig stark. 
Da für diese Störungen des Gefälles keinerlei Hinweise auf tektonische Einflüsse vorliegen, erschien der Verdacht begründet, daß durch Deckschichtenüberlagerung eine Terrassenaufgliederung nur vorgetäuscht wird; dies um so mehr, da beide Störungen des Längsgefälles und der Übergang der „tieferen Fläche“ in die „höhere Fläche“ im Gebiet des eingangs beschriebenen Geländerückens liegen.

Die gleiche Skepsis drängt sich auch gegenüber dem ungewöhnlichen, mäanderartigen Verlauf der Uferlinie der KMT auf, der nicht so recht in das Bild der in der Regel gradlinig oder sehr weit geschwungen verlaufenden Uferränder dieses Raumes passen will, zumal sich keine plausible Erklärung für die Anlage dieser engen Bögen findet.

\section{Einfluß der Deckschichten auf die Gestaltung der Terrassenlandschaft}

\section{Morphologische Kriterien}

In den bisherigen Bearbeitungen ist den Deckschichtenmächtigkeiten bei den morphologischen Terrassengliederungsversuchen so gut wie keine Beachtung zugekommen, obwohl seit FLIEGEL (1908) Lößmächtigkeiten bis zu $10 \mathrm{~m}$ auf den Mittelterrassen dieses Raumes bekannt sind. Eine Ausnahme bildet hier lediglich die Terrassengliederung im Mittelterrassenbogen von Niederaußem (Breddin 1958 und Heller \& Brunnacker 1966).

Die Vermutung, daß der Geländerücken nördlich des Frechener Baches von außerordentlich mächtigen Deckschichten verursacht wird - im südlichen Abschnitt der UMT liegen die durchschnittlichen Lößmächtigkeiten zwischen nur 1,5-3 m - bestätigte sich in zwei tieferen, kurzzeitig offenen Baugruben in Köln-Junkersdorf. Beide Aufschlüsse zeigten Lößmächtigkeiten von über $5 \mathrm{~m}$, ohne daß der Schotterkörper erreicht wurde. Die unteren Partien der Lößprofile waren durch eine geringe Sandkomponente gekennzeichnet.

Wenn man die Deckschichten im Bereich des Geländerückens bis auf das Niveau der Terrassenfläche der UMT - hier bei ca. $+57 \mathrm{~m} \mathrm{NN}$ - abdeckt (vgl. Kap. II. 1.), was die hier beobachteten Lößmächtigkeiten durchaus gestatten, so zeigt sich, daß die UMT unter Beibehaltung ihres ausgeglichenen Längsgefälles bis auf $+53 \mathrm{~m} \mathrm{NN}$ im nördlichsten Teil des Untersuchungsgebietes ungestört durchzieht.

Die Annahme eines morphologischen Ubergangsbereiches zwischen KMT und UMT im Sinne von QuITzow wäre danach nicht mehr aufrechtzuerhalten. Gleichzeitig wäre damit die Existenz der KMT, zumindest im morphologischen Sinne, in Frage gestellt. Ungeklärt bliebe damit aber vorerst die Stellung der bisher als UMT gedeuteten „höheren Fläche" im nördlichen Abschnitt.

Auffälligerweise findet nun aber der Geländerücken nördlich des Frechener Baches seine höhenmäßige Fortsetzung (entlang der $60 \mathrm{~m}$ Isohypse) in dieser „höheren Fläche“: zunächst in dem Geländevorsprung, der den doppelbogigen Verlauf des bisher als Uferrand der KMT aufgefaßten Geländeabfalls bedingt und daran anschließend in den weiten Flächen dieser Geländestufe nordöstlich von Brauweiler.

Die sich dann stellende Frage, ob nicht auch die „höhere Fläche“ eine nur durch besonders mächtige Deckschichten vorgetäuschte Terrassenstufe darstellt, ließ sich durch Tagesaufschlüsse allein nicht klären. Außer der Ziegeleigrube von Brauweiler mit Lößmächtigkeiten um ca. $5 \mathrm{~m}$ standen keine weiteren Profilanschnitte zur Verfügung.

Hier bot sich mit einiger Aussicht auf Erfolg die Auswertung der aus dem Gebiet westlich von Köln vorhandenen zahlreichen Bohrungen an. 


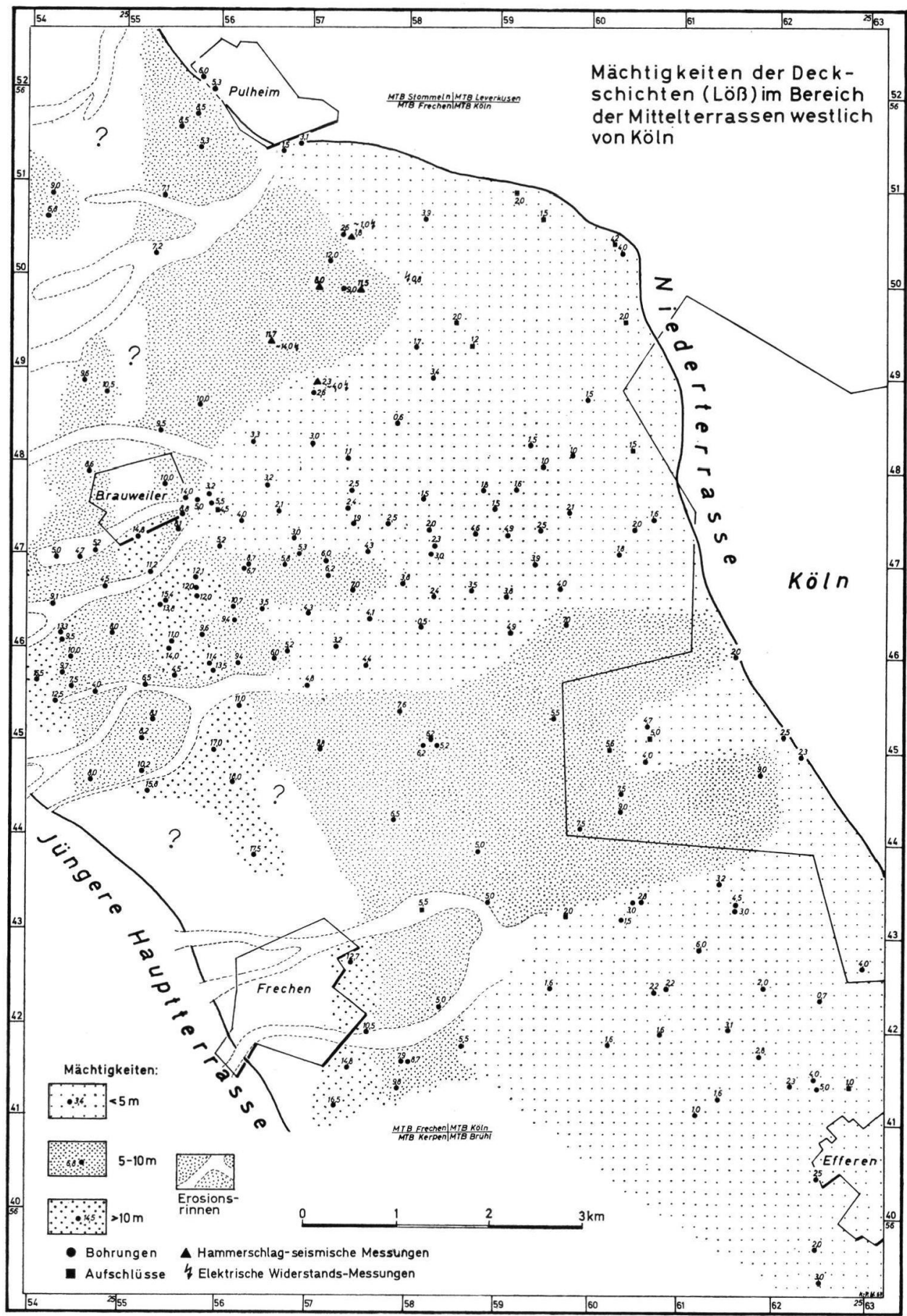

Abb. 3. Mächtigkeiten der Deckschichten (Löß) im Bereich der Mittelterrassen westlich von Köln. 


\section{Ergebnissevon Bohrungen und geophysikalischen Messungen}

Das engmaschige Netz der hier niedergebrachten Bohrungen gibt einen guten Einblick in die Mächtigkeitsverhältnisse der Deckschichten. ${ }^{2}$ )

Nur in den nordwestlich von Brauweiler gelegenen Geländeabschnitten waren zusätzliche Untersuchungen zur Kenntnis der Tiefenlage der Grenzfläche Schotterkörper/Deckschichten notwendig. Hier standen nur zwei Bohrungen zur Verfügung, deren Ergebnisse mit 10 bzw. $12 \mathrm{~m}$ Lößlehm zunächst wenig wahrscheinlich erschienen.

Die daraufhin über dieses Gebiet gelegten refraktionsseismischen Profile und geoelektrischen Widerstandssondierungen (WINTER 1968) bestätigten jedoch diese Angaben. Auch hier lagen die ermittelten Deckschichtenmächtigkeiten zwischen 9 bis $12 \mathrm{~m}$.

Der aus den gewonnenen Daten entworfene Deckschichten-Mächtigkeitsplan (Abb. 3) zeichnet deutlich das morphologische Bild nach.

Die „tiefere Fläche“ (bislang KMT) des nördlichen Abschnitts zeigt ebenso wie die stromaufwärts im südlichen Abschnitt gelegenen Teile der UMT gleichmäßige Lößmächtigkeiten zwischen 2 bis $3 \mathrm{~m}$. Größere Werte werden nur in kleineren, aufgefüllten Reliefdepressionen erreicht.

Dagegen ist eine auffällige sprunghafte Zunahme der Lößmächtigkeiten im Gebiet des Geländerückens nördlich des Frechener Baches zu beobachten. Hier steigen die Werte bis auf $12 \mathrm{~m}$ an, nach den mit einigen Vorbehalten zu betrachtenden Werten der geoelektrischen Widerstandssondierungen sogar auf $14 \mathrm{~m}$ und mehr.

Es bestätigte sich also die Vermutung des Verfassers, daß die von QuiTzow u. a. der UMT zugerechnete „höhere Fläche“ nur eine durch überhöhte Deckschichten vorgetäuschte Terrassenstufe ist.

Der durch noch höhere Lößmächtigkeiten gekennzeichnete Bereich entlang der Linie Frechen-Brauweiler zeichnet den Terrassenhang der OMT nach, der, genau wie der Terrassenhang der Hauptterrasse, durch Lößlehm stark maskiert ist.

Die außerordentlich mächtigen Deckschichten können durch zwei verschiedene Sedimentationsvorgänge erklärt werden. Zunächst kamen auf der UMT vorzugsweise aus dem Bereich der Hauptterrassen abgeschwemmte Lösse, die mit Terrassensanden vermischt sind, schwemmfächerartig zur Ablagerung. Der Sandanteil ist in den kalkfreien Schwemmlössen in der Regel diffus verteilt, nur selten ist er in Schmitzen angereichert. Mit Mächtigkeiten von $6 \mathrm{~m}$ und darüber stellen diese Abschwemmassen den Hauptanteil der Deckschichtenkappe dar. Ihre talwärtige Verbreitung ist in erster Linie erosiv durch die aus der Hauptterrasse einziehenden Nebengewässer bestimmt. Der zweite Sedimentationsvorgang ist die eigentliche Lößanwehung, die mit durchschnittlich 2 bis $3 \mathrm{~m}$ Mächtigkeit die Abschwemmassen und die davor liegenden Schotterfluren überlagerte.

Die endgültige morphologische Gestaltung der Deckschichtenkappe durch jüngere Erosionstätigkeit wird besonders in der südlichen Begrenzung des Geländerückens durch den Frechener Bach und durch die Nahtrinne nordöstlich von Brauweiler deutlich (Abb.2).

\section{Ausbildung der Schotteroberfläche}

Zeigen schon die morphologischen Untersuchungen und der Mächtigkeitsplan, daß die bisher als KMT aufgefaßte Terrassenflur nördlich Köln nur die Fortsetzung der UMT des südlichen Abschnitts darstellt, und daß außerdem die bisher als UMT gedeutete Gelände-

2) Für das gesamte Arbeitsgebiet stehen annähernd 500 Schichten-Verzeichnisse von Flachbohrungen zur Verfügung. Die Unterlagen sind zum großen Teil im Geologischen Landesamt Nordrhein-Westfalen zugänglich. 
stufe nur eine Terrasse vortäuscht, so wird dieses Bild durch die Ausbildung der von Deckschichten entblößten Schotterterrasse verdeutlicht (Abb. 4).

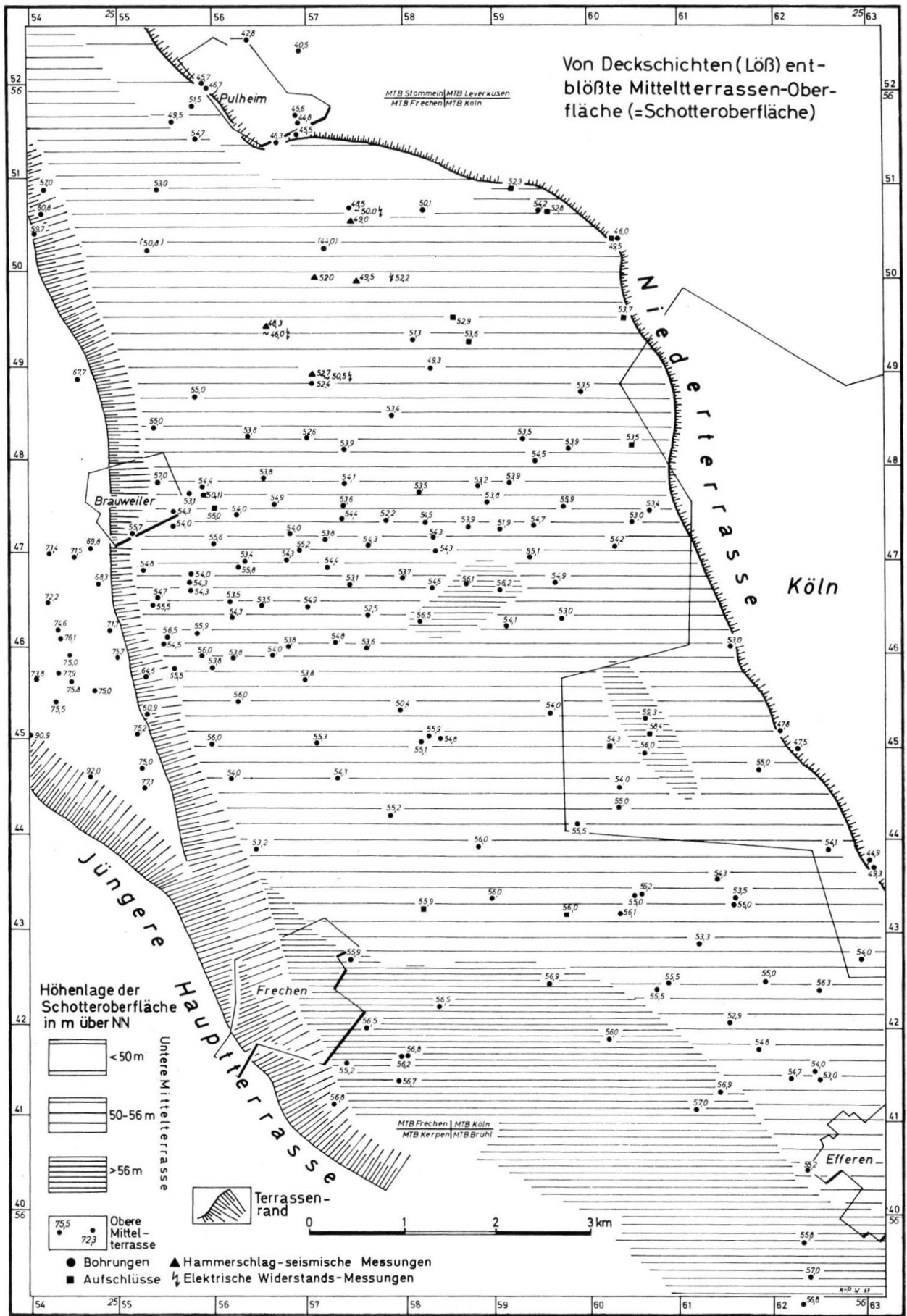

Abb. 4. Von Deckschichten (Löß) entblößte Mittelterrassen-Oberfläche (= Schotteroberfläche). 
Die UMT nimmt im nördlichen Abschnitt, wie auch schon zwischen Bonn und Köln, mit einem ausgeglichenen Längsgefälle die ganze Breite dieser Talstufe vom Abfall zur Niederterrasse bis zum Terrassenhang der OMT ein. Es finden sich auch hier keine Hinweise für die Ausbildung einer tieferen Mittelterrasse.

Damit ist auch die von Tноме $(1958,1959)$ für diesen Raum in Form einer Erosionsterrasse angenommene Verbreitung der KMT unbegründet. Tноме (1958) vermutete, ausgehend von der von QuiTzow (1956) beschriebenen Vereinigung von KMT und UMT in der Höhe von Köln, daß hier die UMT - bedingt durch einen vom Inlandeis („Düsseldorfer Lobus“) verursachten Hochwasserrückstau - zunächst überhöht aufgeschüttet wurde und mit beginnendem Eisrückzug bis auf das Niveau der KMT (= „tiefere Fläche“, Abb. 2) erodiert wurde.

Die bei Pulheim (nördlich Köln) in den Schotterfluren der UMT vereinzelt auftretenden Eintiefungen zwischen 2 bis $3 \mathrm{~m}$, die BredDIN (1958) in Anlehnung an Quitzow (1956) zur Annahme des Vorhandenseins der KMT veranlaßten, sind als Erosionsrinnen aufzufassen, die erst nach Ausbildung der Schotterflur angelegt wurden.

\section{Sedimentologische und pedologische Verhältnisse}

Die südlich Köln von Quitzow (1956) angenommene Überlagerung der UMT durch Aufschüttungen der KMT wurde in der Folge durch PAAs (1962) gestützt.

PaAs stellt weite Teile der uferrandferneren Bereiche der UMT zwischen Bonn und Köln zur KMT (Abb. 1) und betont, daß die UMT hier zwei verschiedenaltrige Schotterkörper (UMT und KMT) enthält. Das Kriterium für diese Untergliederung der fluviatilen Aufschüttung ist für PAAs der von ihm für die UMT als typisch beschriebene Interglazialboden. Profile der UMT, an denen eine Überprägung der Schotter durch diese Bodenbildung nicht nachzuweisen war, stellt er in Analogie zu KMT-Profilen des Krefelder Raumes - dort war dieser Interglazialboden nicht nachzuweisen - zur KMT. Der Schwierigkeit einer solchen Deutung - die UMT bildet hier eine morphologische Einheit (vgl. Kap. II.1.) - begegnet er mit der wenig überzeugenden Argumentation, daß einer teilweisen Erosion der UMT (mindestens $10 \mathrm{~m}$ ) in ihren randlichen Teilen und damit der Ausräumung des Interglazialbodens eine erneute Aufschotterung folgte, die dann genau das ursprüngliche Niveau der UMT erreichte.

Die einzige von PAAs (1962) als Beispiel für diese Schlußfolgerungen beschriebene Lokalität ist ein Profil östlich von Alfter bei Bonn, auf das noch speziell eingegangen wird.

Nach den hier durchgeführten Untersuchungen zur sedimentologischen Entwicklung der UMT kann die Frage einer möglichen Überlagerung der UMT durch Aufschüttungen einer nächst jüngeren Terrasse südlich Köln vom sedimentologischen Aufbau der Schotterprofile her klar verneint werden.

Die Aufschüttungen der UMT sind im Südteil der Niederrheinischen Bucht durch einen rhythmischen Wechsel von mächtigen Grob- und Feinschotter-Horizonten gekennzeichnet (WINTER 1968). Diese Horizonte sind über den gesamten Raum von Profil zu Profil zu verfolgen. Dadurch ist ein direkter profilmäßiger Vergleich der uferrandnahen oder weiter stromaufwärts gelegenen Profile, die sicher zur UMT gehören (Profile: 3, 4, 5, $6,7,8,9,10,11)$, mit den uferrandferneren Profilen, die nach PAAs im Bereich der KMT liegen (Profile: 1, 2, 12, 13, 14), möglich. (Profilverzeichnis s. S. 171. Lage der Profile vgl. Abb. 5.)

An keinem der fraglichen KMT-Profile lassen sich Spuren einer Erosion oder sonstige Hinweise finden, die für die von PAAs geforderte Ausräumung ganzer Teile der UMT sprächen. Die rhythmische Grob-Feinschotter-Wechselfolge ist hier in der gleichen Vollständigkeit und ungestört entwickelt, wie in allen anderen UMT-Profilen dieses Raumes. 
Die hangenden Partien dieser Profile (Horizonte A und B, Winter 1968), die im Sinne von PAAS die KMT repräsentieren müßten, zeigen nach den Ergebnissen der sedimentpetrographischen Analysen (Quarz/Buntschotter-Verhältnis, Korngrößenverteilung, Rundungsgradentwicklung) die gleiche Ausbildung, wie sie für alle anderen UMT-Profile ermittelt wurde.

Letzteres gilt auch für die im nördlichen Abschnitt gelegenen und bisher als Ablagerungen der KMT gedeuteten Schotterserien im Profil der Ziegeleigrube Brauweiler (Profil 17) und in Kiesgruben südöstlich von Pulheim (Profile 15, 16, 18). gelten.

Als ein Beispiel kann das auch von PAAS (1962) beschriebene Profil östlich Alfter bei Bonn

Hier wird der oberste Grobschotter-Horizont durch eine 2-3 m mächtige, vorwiegend sandige Abfolge überlagert, auf die wiederum eine bis $\mathrm{zu} 1,5 \mathrm{~m}$ mächtige Schotterpartie folgt. Diese Schotterlage ist durch einen braunlehmartigen Boden überprägt. Das Profil wird von einer geringmächtigen Lößkappe abgeschlossen.

PAAs deutete dieses Profil als zur KMT gehörig und vermutet in der sandigen Abfolge eine Hochflutbildung der KMT, in der abschließenden Schotterpartie eine Einschwemmung aus OMT und HT.

Die Schotteranalyse zeigt für diese Kiese aber das für die UMT typische Geröllspektrum mit durchschnittlichen Quarzzahlen um 31\%. Nach den vorliegenden Untersuchungen (WINTER 1968) lassen sich seitliche Materialzufuhren aus der OMT und der jHT für den Bereich der UMT zwischen Bonn und Köln recht sicher fassen. Die Quarzzahl steigt dann auf 40\% und darüber hinaus an. Eine seitliche Einschwemmung scheidet danach hier aus. Der gesamte Profilaufbau entspricht der typischen Entwicklung der UMT dieses Raumes.

Die die oberste Schotterlage überprägende Bodenbildung, die von PAAS als ein von OMT und HT her umgelagerter braunlehmartiger gelblichroter Interglazialboden beschrieben wird, läßt sich dann auch zwanglos mit der sonst auf der UMT entwickelten Bodenbildung vergleichen.

Auch die bisher vor allem durch Zonneveld (1956) und Vinken (1959) aus dem Südteil der Niederrheinischen Bucht bekannten Ergebnisse von Schwermineraluntersuchungen geben keinen Anlaß, neben der UMT eine nächst jüngere Mittelterrassen-Aufschüttung anzunehmen.

Im gleichen Sinne sprechen auch die pedologischen Verhältnisse. In allen hier bearbeiteten Profilen sind die oberflächennahen Schotterpartien durch eine Bodenbildung überprägt, die in ihrer Intensität und Mächtigkeit allerdings stärkeren Schwankungen unterliegt. In Übereinstimmung mit PAAs (1962) kann diese Bodenbildung nach ihrer Entwicklung als ein die UMT überprägender Interglazialboden aufgefaßt werden.

PAAS weist darauf hin, daß in einigen nahe zur Niederterrasse gelegenen Profilen (z. B. Profile bei Brühl und Efferen) dieser Boden nicht mehr erhalten ist. ${ }^{3}$ ) Diese Profile stellt er entsprechend der oben angeführten Argumentation zur KMT.

Nach den hier aufgezeigten Kriterien ist dieser Schluß nicht zu rechtfertigen. Ist diese Bodenbildung in einigen uferrandfernen Profilen nicht erhalten, so ist dieser Umstand einfach dadurch zu erklären, daß hier in unmittelbarer Nähe des Abfalls der Ville eine flächenhafte oder auch nur lokal wirksame Abspülung und Ausräumung der obersten Profilabschnitte der UMT stattfand. Dafür spricht auch die von PAAs (1962) für UMTProfile im Raum zwischen Bonn und Köln mitgeteilte Beobachtung, daß diese Bodenbildung immer stark gekappt ist.

Zusammenfassend ergeben die hier vorgelegten Ergebnisse, daß die Krefelder Mittelterrasse im Gegensatz zur bisherigen Auffassung im Südteil der Niederrheinischen Bucht $\mathrm{n}$ ich t ausgebildet ist. Abb. 5 zeigt das revidierte Bild der Terrassenabfolge.

3) Ob die hier untersuchten Profile bei Brühl und Effern den von PaAs (1962) beschriebenen Lokalitäten entsprechen, ist durch die Anlage einer Reihe neuer Aufschlüsse in diesen Gebieten nicht gesichert. 


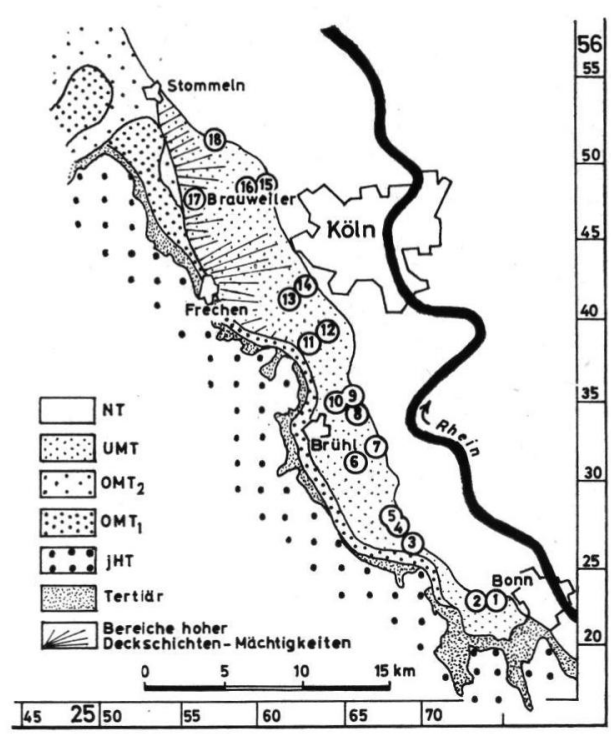

Abb. 5. Verbreitung der UMT im linksrheinischen Teil der Niederrheinischen Bucht mit Lage der bearbeiteten Profile.

A ufschlußverzeichnis

$\begin{array}{cll}\text { Profile } & \text { Blatt Bonn 5208 } & \\ 1 & \text { R: 257304, H: } 562275 & \text { Kiesgrube südöstl. Alfter } \\ 2 & \text { R: 257212, H: } 562324 & \text { Kiesgrube östl. Alfter } \\ & \text { Blatt Sechtem 5207 } & \\ 3 & \text { R: 256885, H: } 562616 & \text { Kiesgrube nördl. Bornheim } \\ 4 & \text { R: 256836, H: } 562775 & \text { Kiesgrube südöstl. Sechtem } \\ 5 & \text { R: 256822, H: } 562815 & \text { Kiesgrube östl. Sechtem } \\ & \text { Blatt Brühl 5107 } & \\ 6 & \text { R: 256647, H: } 563035 & \text { Kiesgrube am Dickopshof } \\ 7 & \text { R: 256760, H: } 563130 & \text { Kiesgrube westl. Keldenich } \\ 8 & \text { R: 256607, H: } 563490 & \text { Kiesgrube nördl. d. Langenackerhofes } \\ 9 & \text { R: 256575, H: } 563540 & \text { Kiesgrube südl. d. Engeldorferhofes } \\ 10 & \text { R: 256520, H: } 563520 & \text { Kiesgrube westl. d. Engeldorferhofes } \\ 11 & \text { R: 256343, H: } 563787 & \text { Ziegeleigrube Kalscheuren } \\ 12 & \text { R: 256495, H: } 563857 & \text { Kiesgrube westl. d. Konraderhofes } \\ 13 & \text { R: 256429, H: } 563965 & \text { Kiesgrube südl. Efferen } \\ 14 & \text { R: 256432, H: } 564000 & \text { Kiesgrube westl. Efferen } \\ & \text { Blatt Köln 5007 } & \\ 15 & \text { R: 255950, H: } 565054 & \text { Kiesgrube nordwestl. Mengenich } \\ 16 & \text { R: 256305, H: } 564928 & \text { Kiesgrube nordöstl. Widdersdorf } \\ & \text { Blatt Frechen 5006 } & \\ 17 & \text { R: 255608, H: } 564775 & \text { Ziegeleigrube Brauweiler } \\ 18 & \text { Blatt Stommeln } 4906 & \\ \text { R: 255438, H: } 565570 & \text { Kiesgrube südl. Stommeln. }\end{array}$

Literaturverzeichnis

Ahorner, L.: Untersuchungen zur quartären Bruchtektonik der Niederrheinischen Bucht. - Eiszeitalter u. Gegenwart, 13, 24-105, OOhringen 1962.

Bertsch, K.; Steeger, A. \& Steusloff, U.: Fossilführende Schichten in der sogenannten Krefelder Mittelterrasse. - Sitz.-Ber. Versamml. niederrhein. geol. Ver., 23 (f. 1929), 1-20, Bonn 1931. 
Breddin, H.: Die unterirdische Oxydation der Braunkohle im Kölner Revier während der Pleistozän-Zeit. - Fortschr. Geol. Rheinld. u. Westf., 2, 683-720, Krefeld 1958.

Fliegel, G.: Erläuterungen zur Geologischen Karte von Preußen, Blatt Frechen. - Berlin 1908.

Heller, F. \& Brunnacker, K.: Halsbandlemming-Reste aus einer Oberen Mittelterrasse des Rheins bei Niederaußem. - Eiszeitalter u. Gegenwart, 17, 97-112, Öhringen 1966.

KaIser, Kh.: Gliederung und Formenschatz des Pliozäns und Quartärs am Mittel- und Niederrhein, sowie in den angrenzenden Niederlanden unter besonderer Berücksichtigung der Rheinterrassen. - In: Köln und die Rheinlande (Festschr. z. XXXIII. Deutsch. Geographentag 1961 in Köln) 236-278, Wiesbaden 1961.

Kopp, E. \& Woldstedt, P.: Über den Charakter der Wärmezeit zwischen Drenthe- und WartheStadial in Norddeutschland. - Eiszeitalter u. Gegenwart, 16, 37-46, Ohringen 1965.

PaAs, W.: Rezente und fossile Böden auf niederrheinischen Terrassen und deren Deckschichten. Eiszeitalter u. Gegenwart, 12, 165-230, OOhringen 1962.

Quitzow, H. W.: Die Terrassengliederung im niederrheinischen Tieflande. - Geol. en Mijnbouw (N. S.), 18, 357-373, 's Gravenhage 1956.

Steeger, A.: Landschaftsformen am linken Niederrhein. - Die Natur am Niederrhein, 1, 1-12, Krefeld 1925 (1925a).

- : Das glaziale Diluvium des Niederrheinischen Tieflandes. - Beitr. I.-VII. Ber. Versamml. niederrh. geol. Ver., f. 1923, 1-46, Bonn 1925 (1925b).

- : Das glaziale Diluvium des Niederrheinischen Tieflandes. - Beitr. VIII.-Ber. Versamml. niederrhein. geol. Ver., f. 1925, 48-60, Bonn 1926.

Tноме, K. N.: Die Begegnung des nordischen Inlandeises mit dem Rhein. - Geol. Jb., 76, 261-308, Hannover 1958.

- : Eisvorstoß und Flußregime am Niederrhein und Zuider See im Jungpleistozän. - Fortschr. Geol. Rheinld. u. Westf., 4, 197-246, Krefeld 1959.

VINKEN, R.: Sedimentpetrographische Untersuchungen der Rheinterrassen im östlichen Teil der Niederrheinischen Bucht. - Fortschr. Geol. Rheinld. u. Westf., 4, 127-170, Krefeld 1959.

Winter, K.-P.: Die Untere Mittelterrasse im Südteil der Niederrheinischen Bucht. - Sonderveröff. Geol. Inst. Univ. Köln, 15, Köln 1968.

Zonneveld, J. I. S.: Schwermineralgesellschaften in niederrheinischen Terrassensedimenten. Geol. en Mijnbouw (N. S.), 18, 395-401, 's Gravenhage 1956.

Manuskr. eingeg. 19. 8. 1970.

Anschrift des Verf.: Dr. K.-P. Winter, Geologisch-Paläontologisches Institut der Universität, 6 Frankfurt (Main), Senckenberganlage 32-34. 